\title{
Psychometric evaluation of 'The 25-item Sex after MI Knowledge Test' in a Swedish context
}

\author{
Petra Svedberg PhD, RN (Senior Lecturer) ${ }^{1}$, Ingela Johansson PhD, RN (Assistant Professor) 2,3,4, Sylvie \\ Persson PhD, RN (Associate Professor) ${ }^{5}$, Åsa Roxberg PhD, RN (Associate Professor) ${ }^{5,6}$, Bengt Fridlund PhD, RN \\ (Professor) $^{5,7}$, Amir Baigi PhD (Associate Professor) ${ }^{8}$, David Brunt PhD, RN (Associate Professor) ${ }^{5}$, Carin Alm \\ Roijer PhD, RN (Senior Lecturer) ${ }^{9}$, Dan Malm PhD, RN (Senior Lecturer) ${ }^{7,10}$, Mikael Rask PhD, RN (Associate \\ Professor) ${ }^{5}$ and Ulrica Nilsson PhD, RN (Associate Professor) ${ }^{11}$, the SAMMI-study group \\ ${ }^{1}$ School of Social and Health Sciences Halmstad University, Halmstad, ${ }^{2}$ Division of Nursing Sciences, Department of Medical and Health \\ Sciences, Linköping University, Linköping, ${ }^{3}$ Department of Cardiology, Linköping Heart Centre, Linköping University Hospital, Linköping, \\ Sweden, ${ }^{4}$ Faculty of Health Sciences, Molde University College, Molde, Norway, ${ }^{5}$ School of Health and Caring Sciences, Linnaeus University, \\ Växjö, Sweden, ${ }^{6}$ University College of Haraldsplass, Bergen, Norway, ${ }^{7}$ School of Health Sciences, Jönköping University, Jönköping, ${ }^{8}$ General \\ Practice and Public Health, Halland County Council, Falkenberg, ${ }^{9}$ The Faculty of Health and Society, Malmö University, Malmö, ${ }^{10}$ Department \\ of Cardiology, County Hospital Ryhov, Jönköping and ${ }^{11}$ Centre of Heath Care Sciences Örebro County Council and School of Health and \\ Medical Sciences, Örebro University, Sweden
}

Scand J Caring Sci; 2012; 26; 203-208

\section{Psychometric evaluation of 'The 25-item Sex after MI Knowledge Test' in a Swedish context}

The patients' sexual life after a myocardial infarction is important for his/her quality of life. In spite of this, many patients are in doubt regarding their sex life after a myocardial infarction (MI) and the sexual information received, and counselling from health care providers has been seen to be insufficient. The purpose of this study was to evaluate the psychometric properties of 'The 25-item Sex after MI Knowledge Test' in a Swedish context. A convenience sample was recruited. The scale was translated into Swedish and completed by 79 former patients from The Heart and Lung Patients' National
Association on two occasions, with an interval of 2 weeks. The scale was tested for face and content validity, internal consistency and test-retest reliability. The result in this study indicates that the instrument has good face and content validity and displayed a moderate internal consistency (alpha 0.61). The instrument showed some level of instability in test-retest reliability with $60 \%$ of the items presenting moderate or strong agreement between the test and retest. Further studies that use this instrument in larger and more diverse samples are thus needed.

Keywords: heart disease, The 25-item Sex after Myocardial Infarction Knowledge Test, validity, reliability.

Submitted 29 August 2010, Accepted 10 June 2011

\section{Introduction}

Myocardial infarction (MI) is a major cause of death and disability worldwide and strikes both men and women. This life-threatening event changes the individual's everyday life in a variety of ways $(1,2)$. The patient's sexual function, desire and behaviour, among other psychosocial factors, may be altered after an MI (3-6). When recovering from MI, it is important to retain a good, or least acceptable, well-being that also includes a sexual life without too much emotional stress. Several studies have, however, shown that patients after MI experience a

\section{Correspondence to:}

Petra Svedberg, School of Social and Health Sciences, Halmstad University, SE-301 18 Halmstad, Sweden.

E-mail: petra.svedberg@hh.se changed or reduced sexual desire followed by an altered sexual activity often in combination with an occurrence of anxiety, depression and sexual dysfunction (7-9).

Apart from the problem that many drugs may cause a sexual dysfunction (10-12), there are also many myths and fears concerning the dangers of resuming sexual intercourse after MI $(13,14)$. It has been suggested that patients and their partners fear that sexual activity could be a trigger for further cardiac ischaemia or even re-infarction (15). It is therefore important to dispel all myths and fears and to provide correct information to both the patients and their partners, so they can understand how and to what extent physical activity including sexual activity may influence the risk for the recurrence of MI $(12,16,17)$.The first step is to provide adequate guidelines, information and support to patients and their partners. One of the problems is that many health care professionals lack the competence and skills in how to communicate 
sexual function after MI $(18,19)$ and feel uncomfortable discussing patients' sexual issues (18-20). Another challenge is to explore how knowledgeable the patients are about sexual activity and heart disease (21). Inquiries concerning sexuality are usually experienced as delicate, and questionnaires should mainly be filled in by patients and partners at home where they can feel safe and not disturbed (22). Secrecy and feeling safe are of utmost importance to obtain true psychometric properties of the instrument used.

Validated instruments testing patients' knowledge concerning sexual activity and the risk for the recurrence of MI are uncommon. In 1979, the sex knowledge and attitude test were designed to measure changes in knowledge and attitudes after a course or programme in human sexuality to prepare health care professionals to aid patients with sexual problems (23). This instrument was, however, not tested in patients after MI, and in 2004, Steinke and Swan (24) developed a questionnaire "The 25-item Sex after MI Knowledge Test' to test the level of knowledge in patients after MI. The questionnaire was modelled after an established sexuality and ageing instrument, the Aging Sexual Knowledge and Attitudes Scale (25). The content validity of 'The 25-item Sex after MI Knowledge Test' was assessed by a panel of experts and was piloted by 10 patients after MI. The questionnaire was considered sufficient with a test-retest reliability of 0.81 , and the alpha coefficient was 0.65 on the first test (24). Steinke and Swan measured the knowledge level of patients resuming sexual activity post-MI for the first time, and further testing of the questionnaire was recommended. However, the questionnaire has only been used on an English-speaking group of patients, and it is thus important to investigate whether the questionnaire is also suitable in a Swedish cardiac context and elucidate how the presented information and absorbed knowledge influences the patients' and their partners' sexual relationship. The purpose of this study was thus to evaluate the psychometric properties of 'The 25-item Sex after MI Knowledge Test' in a Swedish context. Two main issues were focused on (i) an investigation of the internal consistency of the Swedish version of the instrument 'The 25-item Sex after MI Knowledge Test' and (ii) an evaluation of the test-retest reliability of the instrument.

\section{Methods}

\section{Design and setting}

The study had a methodological design where the translated version of the questionnaire 'Sex after MI Knowledge Test' was psychometrically tested. This was carried out during spring 2007 at The Heart and Lung Patients' National Association in Sweden, which is a nonprofit organization working for the goal of quality of life for people with heart and lung diseases (26).

\section{Sample and data collection}

A convenience sample of persons was recruited from The Heart and Lung Patients' National Association at ten local meeting places in different areas of Sweden. The intention was to recruit ten respondents at each local occasion. A majority $(80 \%)$ of the members in the Heart and Lung Patients' National Association have an age $>65$ years (26). The inclusion criteria were that the respondents had own experiences of cardiac disease, i.e. MI and lived with a partner.

At each meeting, a member of the nurse research team informed the respondents about the study and asked the respondents who suffered from a cardiac disease and lived with a partner, to fill in the questionnaires on two occasions with a time interval of 2 weeks to be able to investigate the test-retest reliability of the questionnaire. The questionnaires, including a return envelope, were distributed to the respondents after this information. The first questionnaire was filled in directly and was collected by the researchers, and the second was mailed to the researchers.

The final sample consisted of 79 respondents (47 men and 32 women) who agreed to participate and completed the questionnaires (a response rate of 79\%) on both occasions 1 and 2 . The study was performed according to the rules of the World Medical Association declaration of Helsinki (27). The respondents were informed about the purpose and the structure of the study before they gave their informed consent. Participation was voluntary, and the respondents were informed that they could withdraw from the study at any time.

\section{Questionnaire}

The questionnaire 'Sex after MI Knowledge Test' is a 25 -item self-rating questionnaire (Table 1), developed by Steinke and Swan (24) to measure knowledge about resuming sexual activity post-MI. The respondents list their answers in an unforced-choice format as true, false or do not know. Ten items are reverse-scored. Correct responses receive a score of 3 ; incorrect responses receive a score of 1 , while 'do not know' responses receive a score of 2. Scores can range from 25 to 75 , with a higher score indicating greater knowledge. The instrument has previously been assessed for content validity and for reliability (24).

\section{Translation procedure}

The original version of the 'Sex after MI Knowledge Test' was received from the originator and permission to use it was obtained. The Swedish version of the questionnaire was constructed through a translation/back-translation procedure $(28,29)$. This method involved translation from 
Table 1 Items and correct answers in the original instrument 'Sex after myocardial infarction Knowledge Test'

\begin{tabular}{|c|c|}
\hline Items & $\begin{array}{l}\text { Correct } \\
\text { answers }\end{array}$ \\
\hline $\begin{array}{l}\text { 1. A danger sign to report to the physician is shortness of breath or increased heart rate (pulse) for more than } 15 \text { minutes } \\
\text { after intercourse }\end{array}$ & A \\
\hline 2. Drinking alcohol prior to sex will help you relax and improve sex & B \\
\hline 3. Some medicines used for high blood pressure, anxiety or depression can affect sex & A \\
\hline 4. You should try not to upset your partner with your fears about resuming sex & B \\
\hline 5. Palpitations (rapid heart beating) lasting more than 15 minutes after intercourse are normal & B \\
\hline 6. Sex can generally be safely resumed within a few weeks after the heart attack & A \\
\hline 7. It is helpful to be rested before intercourse & A \\
\hline 8. Masturbation and oral sex are more harmful to the heart than sexual intercourse & B \\
\hline 9. You should choose your usual position for sex or one that is most comfortable and that does not tire you & A \\
\hline 10. If you think a medicine is causing a problem with sex, you should stop it immediately & B \\
\hline 11. A common emotional reaction after heart attack is depression & A \\
\hline 12. A normal response during sex is an increased heart rate, blood pressure and rate of breathing & A \\
\hline $\begin{array}{l}\text { 13. A good way to ease back into sex is to talk with your partner about your feelings about the heart attack while taking } \\
\text { a daily walk }\end{array}$ & A \\
\hline 14. It is important to have sex as often as before your heart attack & B \\
\hline 15. Late evening or the end of the day is the best time to have sex when you are more relaxed & B \\
\hline 16. Sexual foreplay when you are more relaxed puts less strain on your heart & A \\
\hline $\begin{array}{l}\text { 17. A hotel room is the ideal place to resume sex after a heart attack in order to create a more exiting } \\
\text { atmosphere }\end{array}$ & B \\
\hline 18. If you have chest pain during sex, you should stop and rest & A \\
\hline 19. Anal intercourse can be resumed just as vaginal intercourse since it has less effects on the heart & B \\
\hline 20. Not being able to sleep after intercourse or extreme fatigue the day after intercourse is normal & B \\
\hline 21. Wait 2-3 hours after a heavy meal before having sex & A \\
\hline 22. It is normal to feel angry or helpless if your partner is overprotective of you after a heart attack & A \\
\hline 23. You should report to your physician a feeling of tightness, fullness or chest pain during sex & A \\
\hline 24. A room temperature that is not to hot or cold is important for sex & A \\
\hline 25. If you are tense or tired, you should not have intercourse until after a good night's sleep & A \\
\hline
\end{tabular}

Answers alternative: A. True B. False C. Don't know.

English to Swedish and back again by two bilingual health care professionals and researchers, independently of each other. First one of the authors (MR), a native Swedish speaker fluent in both English and Swedish, translated the scale to Swedish, and then another author (DB), a native English speaker also fluent in both languages, with no previous knowledge of the original scale, re-translated it into English. Possible transcultural differences were also discussed among the nurse research team during this process with the aim of making the instrument culturally equivalent to promote a sound content validity. In addition, a midwife with long experience as a sexologist was involved throughout the whole translation procedure. Differences between the original version and the re-translated version were then discussed in the research team, consisting of members very familiar in cardiovascular care to improve the quality of the Swedish translation $(30,31)$. The relevance, readability and clarity of the items of the Swedish version was discussed in the research team as well as with the midwife and found to be adequate.

\section{Statistical analysis}

The questionnaire was examined for content validity, internal consistency and test-retest reliability. With regard to face and content validity, the respondents who completed the questionnaire were asked to review the questions for relevance, clarity and readability as suggested by Polit and Beck (30). Cronbach's alpha was used to investigate the internal consistency for the complete instrument, and the Cronbach's alpha coefficient was recommended as acceptable if alpha was $\geq 0.70$ (31). Intraclass correlation coefficients (ICC) were calculated for each item in the instrument to investigate test-retest reliability. The time interval between the first and the second occasion was 2 weeks in accordance with recommendations from Streiner and Norman (32). The ICC produces a value of 1.0 only when the scores on the first occasion are exactly the same as those on second occasion. Guidelines used for interpretation of ICCs were based on studies demonstrating that for ordinal data, ICCs are mathematically equivalent to the weighted kappa statistic $(33,34)$. 
ICC values $<0.20$ indicate poor agreement, 0.21-0.40 indicate fair agreement, $0.41-0.60$ indicate moderate agreement, $0.61-0.80$ indicate strong agreement and finally $>0.8$ indicate almost perfect agreement (35). Statistical analyses were performed using the sPss software 15.0 (SPSS Inc., Chicago, IL, USA).

\section{Results}

The questionnaire was adjudged by the respondents to be relevant for the focus of the study as well as having sufficient clarity and readability, and they found it relevant to complete. The Cronbach's alpha coefficient for the complete instrument of 25 items included was equal for both the first and the second occasion (0.610 vs. 0.615$)$. The ICC values for each item on test-retest varied according to the items considered and ranged between 0.117 and 0.658 (Table 2). One of the items (4\%) showed poor agreement, nine items $(36 \%)$ showed fair agreement, eleven items (44\%) showed moderate agreement and finally four items (16\%) showed strong agreement between the two occasions.

\section{Discussion}

The results in the present study indicate that the instrument 'Sex after MI Knowledge Test' had good face and

Table 2 Intraclass correlation coefficients (ICC) for the original instrument 'Sex after myocardial infarction Knowledge Test' $(n=79)$

\begin{tabular}{|c|c|c|c|}
\hline Item & $n$ & ICC & $95 \% \mathrm{Cl}$ \\
\hline 1. & 75 & 0.411 & $0.20-0.58$ \\
\hline 2. & 75 & 0.560 & $0.38-0.70$ \\
\hline 3. & 75 & 0.419 & $0.21-0.59$ \\
\hline 4. & 74 & 0.610 & $0.44-0.73$ \\
\hline 5. & 75 & 0.496 & $0.30-0.65$ \\
\hline 6. & 73 & 0.353 & $0.13-0.54$ \\
\hline 7. & 75 & 0.117 & $-0.11-0.33$ \\
\hline 8. & 75 & 0.495 & $0.30-0.65$ \\
\hline 9. & 75 & 0.325 & $0.11-0.51$ \\
\hline 10. & 74 & 0.427 & $0.22-0.60$ \\
\hline 11. & 73 & 0.375 & $0.16-0.56$ \\
\hline 12. & 75 & 0.355 & $0.14-0.54$ \\
\hline 13. & 75 & 0.358 & $0.14-0.54$ \\
\hline 14. & 75 & 0.658 & $0.51-0.77$ \\
\hline 15. & 75 & 0.535 & $0.35-0.68$ \\
\hline 16. & 75 & 0.425 & $0.22-0.59$ \\
\hline 17. & 74 & 0.642 & $0.48-0.76$ \\
\hline 18. & 75 & 0.439 & $0.24-0.60$ \\
\hline 19. & 75 & 0.339 & $0.12-0.52$ \\
\hline 20. & 75 & 0.359 & $0.14-0.54$ \\
\hline 21. & 74 & 0.644 & $0.49-0.76$ \\
\hline 22. & 74 & 0.365 & $0.15-0.55$ \\
\hline 23. & 74 & 0.592 & $0.42-0.72$ \\
\hline 24. & 73 & 0.523 & $0.33-0.67$ \\
\hline 25. & 73 & 0.306 & $0.08-0.50$ \\
\hline
\end{tabular}

content validity and displayed a moderate internal consistency and some level of instability in test-retest reliability. In the present study, a convenience sample of persons was recruited from The Heart and Lung Patients' National Association at 10 local meeting places in different areas of Sweden. A limitation of this study could be the highly selective sample that was requested to complete the instrument. First, delegates visiting a local meeting arranged by The Heart and Lung Patients' National Association might not be representative of the majority of MI patients, thereby making it difficult to answer the relevance of the questions in an adequate manner. However, the respondents posed that the questionnaire was relevant, clear and easy to read and complete. Second, a majority of the respondents were older than 65 years of age, which is probably slightly above the average for those suffering from their first MI. Third, the sample size of 79 responders (79\%) could be considered as low. Sexual issues are often seen as being of a sensitive nature and a 'taboo' in our society, and survey questions about sexual behaviours are known to be classified as embarrassing by survey methodologists (36), which may be one explanation for the affected response rate.

Translation is a critical part in cultural adjustments of instruments as the meaning can be changed across languages (29). It is indispensible to undertake a systematic process to ensure validity of translated questionnaires, and we do not believe that any essential nuances are lost through the translation/back-translation procedure. We have established that the proposed survey questionnaire is translationally and culturally equivalent. The questionnaire is considered to be appropriate in a Swedish context because there was no major discrepancy encountered between the two translators, both highly fluent in English and Swedish. Furthermore, consultation with the research team, who had long experience of cardiovascular care, and the midwife, who had long experience in sexology, was also beneficial in the translation procedure as well as for the relevance, readability and clarity of the items.

Face and content validity for the Swedish version of the 'Sex after MI Knowledge Test' was supported by the fact that the items were assessed to be adequate by the experts in the research group and the respondents in terms of the relevance and the focus of the study. Because the instrument is a brief assessment scale of knowledge about sex after MI and is easy to respond to, then it may be considered to be a user-friendly instrument.

The reliability of the 'Sex after MI Knowledge Test' can be considered moderate because of the fact that homogeneity showed a Cronbach's coefficient alpha of 0.61 for both the first and the second occasion for the complete questionnaire. This is quite similar to the first test of Cronbach's alpha by Steinke and Swan (24), which showed an alpha coefficient of 0.65 . It is recommended that Cronbach's coefficient alpha should exceed 0.70 for a 
developing questionnaire and 0.80 for a more established questionnaire (37). The stability of the scale over time with a 2-week span that is deemed to be suitable for assessing the stability of a questionnaire (32) showed that the ICC ranged between 0.117 and 0.658 and that $60 \%$ of the items presented moderate or strong agreement between the test and retest. The item 'it is helpful to be rested before intercourse' appears in particular to have low test-retest reliability. One explanation can be that in Steinke and Swan's study reporting the development of the instrument (24), the patients viewed a video including information about sexual activity after a MI and thereafter tested the level of knowledge. Our study did not include any video, and when asking about knowledge, the respondents on the first occasion answer questions about the statement, while on the second occasion, their answer includes a reflection on the statement, which shows an increase in knowledge. Another explanation for the moderate stability could be that the respondents have poor overall knowledge and have not received adequate information and support from the health care professionals about sexuality in relation to the cardiac disease. This is supported by Lunelli et al. (17) who found that $60 \%$ of the patients were in doubt regarding their sex life following hospital discharge and that receiving sexual information and counselling from health care professionals are reported as being at a very low level by both patients and nurses $(17,25,38)$. The attitudes towards sexuality can be affected by cultural barriers, and talk about sexuality can also be considered as 'taboo' from many people. Even in Sweden, health care professionals still feel uncomfortable discussing patients' sexual issues and how to communicate sexual function after MI (25). To use a questionnaire exploring how knowledgeable the patients as well as the partner are about sexual activity and heart disease can be a viable tool in health care and a personal support for the couple in their recovery after an MI event. These moderate findings about the homogeneity and stability reliability indicate the need for further investigation.

\section{Conclusions}

Psychometrically tested instruments for measuring knowledge concerning sexual activity on the risk for the recurrence of MI are not common. The present study, which investigated the questionnaire 'Sex after MI Knowledge Test' in a Swedish context, suggested that the questionnaire showed moderate psychometric properties. The findings provide good support for the face and content validity but only moderate support for internal consistency and test-retest reliability. Further studies are therefore needed using this instrument in larger and more diverse samples.

\section{Acknowledgements}

The study was supported by grants from HSF (Council for Medical Health Care Research in South Sweden). We are grateful to the participants for taking part in the study.

\section{Conflict of interest}

None declared.

\section{Author contributions}

The authors are members of the SAMMI project and have taken part in several meetings discussing research topics. All of the authors have contributed in this study with design and instrument development, data collection and interpretation of the results as well as having contributed in writing the manuscript. B. Fridlund originally designed the study, A. Baigi has provided the statistical analysis and P. Svedberg and U. Nilsson supervised it. The manuscript was drafted by P. Svedberg, S. Persson, I. Johansson, C. Alm-Roijer and U. Nilsson. Critical revisions for significant intellectual content were made by the other authors.

\section{References}

1 Sjöström-Strand A, Fridlund B. Stress in women's daily life before and after a myocardial infarction: a qualitative analysis. Scand J Caring Sci 2007; 21 : 10-17.

2 Kristofferzon M-J, Löfmark R, Carlsson M. Managing consequences and finding hope - experiences of Swedish women and men 4-6 month after myocardial infarction. Scand $J$ Caring Sci 2008; 22: 367-75.

3 Arenhall E, Kristofferzon M-L, Fridlund B, Nilsson U. The female partners view of the intimate relationships after a myocardial infarction. J Clin Nurs 2010; 20: 1677-84.

4 Johansson-Sundler A, Dahlberg K, Ekenstam C. The meaning of close relationships and sexuality: women's well-being following a myocardial infarction. Qual Health Res 2009; 19(3): 375-87.

5 Drory Y, Kravetz S, Weingarten M. Comparison of sexual activity of women and men after a first acute myocardial infarction. $A m \mathrm{~J}$ Cardiol 2000; 85: 1283-7.

6 Steinke EE, Wright DW. The role of sexual satisfaction, age and cardiac risk factors in the reduction of postMI anxiety. Eur J Cardiovasc Nurs 2006; 5(3): 190-6.

7 Kazemi-Salem D, Pishgou B, Assari S, Tavallaii SA. Fear of sexual intercourse in patients with coronary artery disease: a pilot study of associated morbidity. J Sex Med 2007; 4(6): 1619-25.

8 Mosack V, Steinke EE. Trends in sexual concerns after myocardial infarction. J Cardiovasc Nurs 2009; 24(2): 162-70.

9 Steinke EE, Wright DW, Chung ML, Moser DK. Sexual self-concept, 
anxiety and self-efficacy predict sexual activity in heart failure and healthy elders. Heart Lung 2008; 37: 323-33.

10 Csoka AB, Szyf M. Epigenetic sideeffects of common pharmaceuticals: a potential new field in medicine and pharmacology. Med Hypotheses 2009; 73: 770-80.

11 Do C, Huyghe E, Lapeyre-Mestre M, Montastruc JL, Bagheri H. Statins and erectile dysfunction: results of a case/ non- case study using the French Pharmacovigilance System Database. Drug Saf 2009; 32(7): 591-7.

12 Stein R, Hohmann CB. Sexual activity and the heart. Arq Bras Cardiol 2006; 86(1): 61-67.

13 Steinke EE. Sexual counseling after myocardial infarction. Am J Nurs 2000; 100(12): 38-43; quiz 44.

14 Özdamir L, Akdemir N. Nurses' knowledge and practice involving patients' resuming sexual activity following myocardial infarction; implications for training. Aust $J A d v$ Nurs 2009; 26(1): 42-52.

15 Möller J, Ahlbom A, Hulting J, Diderichsen F, de Faire U, Reuterwall C, Hallqvist J. Sexual activity as a trigger of myocardial infarction. A case-crossover analysis in the Stockholm Heart Epidemiology Programme (SHEEP). Heart 2001; 86: 387-90.

16 Fridlund B. Healthy sexual life after a cardiac event: what do we know and what do we do? Eur J Cardiovasc Nurs 2009; 8(3): 159-60.

17 Lunelli RP, Rabello ER, Stein R, Goldmeier S, Morales MA. Sexual activity after myocardial infarction: taboo or lack of knowledge? Arq Bras Cardiol 2008; 90(3): 156-9.

18 Jaarsma T, Strömberg A, Fridlund B, De Geest S, Mårtensson J, Moons $\mathrm{P}$, Norekval TM, Smith K, Steinke E, Thompson DR; UNITE research group.
Sexual counseling of cardiac patients: nurses' perception of practice, responsibility and confidence. Eur $J$ Cardiovasc Nurs 2010; 9: 24-29.

19 Ivarsson B, Fridlund B, Sjöberg T. Information from health care professionals about sexual function and coexistece after myocardial infarction: a Swedish national survey. Heart Lung 2009; 38: 330-5.

20 Rubin R. Communication about sexual problems in male patients with multiple sclerosis. Nurs Stand 2005; 19(24): 33-37.

21 Potvin L, Richard L, Edwards AC. Knowledge of cardiovascular disease risk factors among the Canadian population: relationships with indicators of socioeconomic status. Can Med Assoc J (CMAJ) 2000; 162 (9 Suppl.): 5-11.

22 Shoemaker P, Eichholz M, Skewes E. Item non-response: distinguishing between don't know and refuse. Int $J$ Public Opinion Res 2002; 14(2): 193 201.

23 Miller WR, Lief H. The sex knowledge and attitude test. J Sex Marital Ther 1979; 5(3): 282-7.

24 Steinke EE, Swan JH. Effectiveness of a videotape for sexual counselling post-MI. Res Nurs Health 2004; 27: 269-80.

25 White CB, Catania JA. Psychoeducation al intervention for sexuality with the aged family members of the aged and people who work with the aged. Int J Aging Hum Dev 1982; 15: 121-38.

26 Swedish Heart and Lung Association. 2009. http://www.hjart-lung.se (last accessed 5 April 2009).

27 World Medical Association Declaration of Helsinki. Ethical Principles for Medical Research Involving Human Subjects. 59th wma general assembly, October 2008, Seoul.
28 Brislin RW. Back-translation for cross-cultural research. J Cross Cultural Psychol 1970; 1(3): 185-216.

29 Cha ES, Kim KH, Erlen JA. Translation of scales in cross-cultural research: issues and techniques. $J \mathrm{Adv}$ Nurs 2007; 58: 386-95.

30 Polit DF, Beck CT. Nursing Research: Generating and Assessing Evidence for Nursing Practice. 2008, Wolters Kluwer Health/Lippincott, Williams \& Wilkins, Philadelphia.

31 Burns N, Grove SK. The Practice of Nursing Research - Conduct, Critique and Utilization, 4th edn. 2001, Saunders Company, Philadephia.

32 Streiner DL, Norman GR. Health Measurement Scales. A Practical Guide to Their Development and Use. 2003, Oxford University Press, Oxford.

33 Landis JR, Koch GG. Measurement of observer agreement for categorical data. Biometrics 1977; 33: 159-74.

34 Fleiss JL, Cohen J. Equivalence of weighted kappa and intraclass correlation coefficient as measures of reliability. Educ Psychol Meas 1973; 33: 613-9.

35 Altman DG. Practical Statistics for Medical Research. 1991, Chapman \& Hall, London.

36 Tourangeau R, Yan T. Sensitive questions in surveys. Psychol Bull 2007; 133(5): 859-83.

37 Rattray J, Jones MC. Essential elements of questionnaire design and development. J Clin Nurs 2007; 16: 234-43.

38 Vassiliadou A, Stamatopoulou E, Triantafyllou G, Gerodimou E, Toulia G, Pistolas D. The role of nurses in the sexual counseling of patients after myocardial infarction. Health Sci J 2008; 2(1): 111-8. 